MSC 20D60, 20B40, 20B35

\title{
ON THE BASE SIZE OF A TRANSITIVE GROUP WITH SOLVABLE POINT STABILIZER 1
}

\section{E.P. Vdovin}

We prove that the base size of a transitive group $G$ with solvable point stabilizer and with trivial solvable radical is not greater than $k$ provided the same statement holds for the group of $G$-induced automorphisms of each nonabelian composition factor of $G$.

Keywords: solvable subgroup, finite simple group, solvable radical.

\section{Introduction}

The term "group" always means a "finite group". We use symbols $A \leqslant G$ and $A \leqslant G$ if $A$ is a subgroup of $G$, and $A$ is a normal subgroup of $G$, respectively. If $\Omega$ is a (finite) set, then by $\operatorname{Sym}(\Omega)$ we denote the group of all permutations of $\Omega$. We also denote $\operatorname{Sym}(\{1, \ldots, n\})$ by $\operatorname{Sym}_{n}$. Given $H \leqslant G$ we denote by $H_{G}=\cap_{g \in G} H^{g}$ the core of $H$.

Let $A, B$ be subgroups of $G$ such that $B \leqslant A$. Then $N_{G}(A / B):=N_{G}(A) \cap N_{G}(B)$ is the normalizer of $A / B$ in $G$. If $x \in N_{G}(A / B)$, then $x$ induces an automorphism of $A / B$ by $B a \mapsto B x^{-1} a x$. Thus there exists a homomorphism $N_{G}(A / B) \rightarrow \operatorname{Aut}(A / B)$. The image of $N_{G}(A / B)$ under this homomorphism is denoted by $\operatorname{Aut}_{G}(A / B)$ and is called a group of $G$-induced automorphisms of $A / B$.

Assume that $G$ acts on $\Omega$. An element $x \in \Omega$ is called a $G$-regular point if $|x G|=|G|$, i.e., if the $G$-orbit of $x$ is regular. Define an action of $G$ on $\Omega^{k}$ by

$$
g:\left(i_{1}, \ldots, i_{k}\right) \mapsto\left(i_{1} g, \ldots, i_{k} g\right)
$$

If $G$ acts faithfully and transitively on $\Omega$, then the minimal $k$ such that $\Omega^{k}$ possesses a $G$-regular point is called the base size of $G$ and is denoted by Base $(G)$. For every natural $m$ the number of $G$-regular orbits in $\Omega^{m}$ is denoted by $\operatorname{Reg}(G, m)$ (this number equals 0 if $m<\operatorname{Base}(G)$ ). If $H$ is a subgroup of $G$ and $G$ acts on the set $\Omega$ of right cosets of $H$ by right multiplications, then $G / H_{G}$ acts faithfully and transitively on $\Omega$. In this case we denote $\operatorname{Base}\left(G / H_{G}\right)$ and $\operatorname{Reg}\left(G / H_{G}, m\right)$ by $\operatorname{Base}_{H}(G)$ and $\operatorname{Reg}_{H}(G, m)$ respectively. We also say that $\operatorname{Base}_{H}(G)$ is the base size of $G$ with respect to $H$. Clearly, $\operatorname{Base}_{H}(G)$ is the minimal $k$ such that there exist $x_{1}, \ldots, x_{k} \in G$ with $H^{x_{1}} \cap \ldots \cap H^{x_{k}}=H_{G}$.

There are a lot of papers dedicated to this subject. It is impossible to mention all of them, since the list of references would be much longer that the paper. We mention the papers, whose results are used in the present article. A.Seress in [8, Theorem 1.1] proved that the base size of a primitive solvable permutation group is not greater than 4. In [4] S.Dolfi proved that in every $\pi$-solvable group $G$ there exist elements $x, y \in G$ such that the equality $H \cap H^{x} \cap H^{y}=O_{\pi}(G)$ holds, where $H$ is a $\pi$-Hall subgroup of $G$ (see also [10]). V.I.Zenkov in [12] constructed an example of a group $G$ with a solvable $\pi$-Hall subgroup $H$ such that the intersection of five subgroups conjugate with $H$ in $G$ is equal to $O_{\pi}(G)$, while the intersection of every four conjugates of $H$ is greater than $O_{\pi}(G)$ (see

\footnotetext{
${ }^{1}$ The author gratefully acknowledges the support from RFBR, projects 10-01-00391 and 10-01-90007, ADTP "Development of the Scientific Potential of Higher School" of the Russian Federal Agency for Education (Grant 2.1.1.419), Federal Target Grant "Scientific and educational personnel of innovation Russia" for 2009-2013 (government contract No. 02.740.11.5191 and No. 14.740.11.0346), Deligne 2004 Balzan prize in mathematics, and the Lavrent'ev Young Scientists Competition (No 43 on 04.02.2010).
} 
Example 9 below). In [11] it is proven that if, for every almost simple group $S$ possessing a solvable $\pi$-Hall subgroup $H$, the inequalities $\operatorname{Base}_{H}(S) \leqslant 5$ and $\operatorname{Reg}_{H}(S, 5) \geqslant 5$ hold, then for every group $G$ possessing a solvable $\pi$-Hall subgroup $H$ the inequality $\operatorname{Base}_{H}(G) \leqslant 5$ holds. In the present paper we prove the following

Theorem 1. Let $G$ be a group and let

$$
\{e\}=G_{0}<G_{1}<G_{2}<\ldots<G_{n}=G
$$

be a composition series of $G$ that is a refinement of a chief series. Assume that for some $k$ the following condition (Orb-solv) holds: If $G_{i} / G_{i-1}$ is nonabelian, then for every solvable subgroup $T$ of $\operatorname{Aut}_{G}\left(G_{i} / G_{i-1}\right)$ we have

$$
\operatorname{Base}_{T}\left(T\left(G_{i} / G_{i-1}\right)\right) \leqslant k \text { and } \operatorname{Reg}_{T}\left(T\left(G_{i} / G_{i-1}\right), k\right) \geqslant 5
$$

Then, for every maximal solvable subgroup $S$ of $G$, we have $\operatorname{Base}_{S}(G) \leqslant k$.

The author of the paper insert to the "Kourovka notebook" [13] the following problem 17.41.

Problem 1. Let $S$ be a solvable subgroup of a group $G$ with $S(G)=\{e\}$.

(a) (L.Babai, A.J.Goodman, L.Pyber) Do there exist 7 conjugates of $S$ such that their intersection is trivial?

(b) Do there exist 5 conjugates of $S$ such that their intersection is trivial?

Theorem 1 reduces both parts of Problem 1 to the investigation of almost simple groups. Notice also that Theorem 1 generalizes the main result of [11] in the following way.

Corollary 2. Let $G$ be a group possessing a solvable $\pi$-Hall subgroup $H$. Assume that for $k=5$ condition (Orb-solv) holds. Then $\operatorname{Base}_{H}(G) \leqslant 5$.

We prove the corollary in Section 3 of the article.

We remark that recently it was proved by T.C.Burness, M.W.Liebeck, E.O'Brien, A.Shalev, R.A.Wilson, etc that if $G$ is a primitive group of almost simple type and the action is not standard, then $G$ has the base size at most 7, answering a conjecture of Peter Cameron (see [2] and the bibliography thereafter). In light of Theorem 1, these results seem to be relevant to a solution of Problem 1 in finite almost simple groups. Nevertheless they cannot be applied immediately since arbitrary solvable subgroup of a symmetric group or of a classical group may lie in a maximal subgroup giving a standard action.

\section{Notation and preliminary results}

By $|G|$ we denote the cardinality of $G$. By $A: B$ we denote a split extension of a group $A$ by a group $B$. For a group $G$ and a subgroup $M$ of $\operatorname{Sym}_{n}$ by $G \imath M$ we always denote the permutation wreath product. We identify $G>M$ with the natural split extension $\left(G_{1} \times \ldots \times G_{n}\right): M$, where $G_{1} \simeq \ldots \simeq G_{m} \simeq G$ and $M$ permutes $G_{1}, \ldots, G_{n}$. Given group $G$, we denote by $S(G)$ the maximal normal solvable subgroup of $G$. We denote by $e$ the identity element of $G$. A group $G$ is called almost simple if there exists a nonabelian simple group $L$ such that $\operatorname{Inn}(L) \leqslant G \leqslant \operatorname{Aut}(L)$.

The following statement is evident. 
Lemma 3. If $S$ is a maximal solvable subgroup of $G$, then $N_{G}(S)=S$.

Lemma 4. [9, Lemma 1.2] Let $H$ be a normal subgroup of a group $G$, and let $(A / H) /(B / H)$ be a composition factor of $\mathrm{G} / \mathrm{H}$.

Then $\operatorname{Aut}_{G}(A / B) \simeq \operatorname{Aut}_{G / H}((A / H) /(B / H))$.

Lemma 5. Let $S$ be a maximal solvable subgroup of $G$ and let $N$ be a normal subgroup of $G$ containing $S(G)$. Then $N_{N}(N \cap S)=N \cap S$.

Proof. Assume that the claim is false and $G$ is a counter example of minimal order. Assume that $S(G) \neq\{e\}$ and consider the natural homomorphism

$$
\text { - }: G \rightarrow G / S(G) \text {. }
$$

Clearly $\bar{S}$ is a maximal solvable subgroup of $\bar{G}$ and $S(\bar{G})=\overline{S(G)}=\{e\}$. Moreover, $|\bar{G}|<|G|$. Since $G$ is a counter example of minimal order it follows that $N_{\bar{N}}(\bar{N} \cap \bar{S})=\bar{N} \cap \bar{S}$. Now $S(G)$ lies in both $N$ and $S$, hence $N_{N}(N \cap S)$ is a complete preimage of $N_{\bar{N}}(\bar{N} \cap \bar{S})=\bar{N} \cap \bar{S}$, and so $N_{N}(N \cap S)=N \cap S$. Thus $S(G)=\{e\}$.

Set $M=N_{G}(N \cap S), L=N_{N}(N \cap S)=N \cap M$. In view of [5, Proposition 3], $N \cap S \neq\{e\}$, so $S(M) \geqslant S \cap N \neq\{e\}$ and $M$ is a proper subgroup of $G$. Clearly $S \leqslant M$, so the maximality of $S$ implies $S(M) \leqslant S$. Moreover $L$ is normal in $M$. So $L S(M)$ is normal in $M$. Since $|M|<|G|$, we obtain

$$
\left.N_{L S(M)}(S \cap L S(M))\right)=S \cap L S(M)=(S \cap L) S(M) \leqslant S .
$$

Now suppose that $x \in L$. We have $N \cap S \leqslant L \leqslant N$, so $L \cap S=N \cap S$. By construction, $L=N_{N}(L \cap S)$, so $L \cap S \preccurlyeq L$. Moreover $L \leqslant M$, hence $x$ normalizes $S(M)$, and so $x$ normalizes $(S \cap L) S(M)=$ $\left.N_{L S(M)}(S \cap L S(M))\right)$, in particular, $x \in S$. Thus $L=S \cap N$ and $G$ is not a counter example.

Assume that a group $G$ possesses a normal subgroup $T$ satisfying the following conditions:

(C1) there exists a nonabelian simple group $L$ such that $T \simeq L_{1} \times \ldots \times L_{k}$ and $L_{1} \simeq \ldots \simeq L_{k} \simeq L$;

(C2) the subgroups $L_{1}, \ldots, L_{k}$ are conjugate in $G$;

(C3) $C_{G}(T)=\{e\}$.

By [6, Satz 12.5, p. 69], $G$ acting by conjugation on $T$ permutes $L_{1}, \ldots, L_{k}$. Condition (C2) implies that $N_{G}\left(L_{1}\right), \ldots, N_{G}\left(L_{k}\right)$ are conjugate in $G$. It follows that $G$ acts on the right cosets of $N_{G}\left(L_{1}\right)$ by right multiplication, let $\rho: G \rightarrow \operatorname{Sym}_{k}$ be the corresponding permutation representation. The action by right multiplication of $G$ on the right cosets of $N_{G}\left(L_{1}\right)$ coincides with the action by conjugation of $G$ on the set $\left\{L_{1}, \ldots, L_{k}\right\}$, and $G \rho$ is a transitive subgroup of $\operatorname{Sym}_{k}$. By [6. Hauptsatz 1.4, p. 413] there exists a monomorphism

$$
\varphi: G \rightarrow\left(N_{G}\left(L_{1}\right) \times \ldots \times N_{G}\left(L_{k}\right)\right):(G \rho)=N_{G}\left(L_{1}\right) \imath(G \rho)=M .
$$

Since $C_{G}\left(L_{i}\right)$ is a normal subgroup of $N_{G}\left(L_{i}\right)$, it follows that $C_{G}\left(L_{1}\right) \times \ldots \times C_{G}\left(L_{k}\right)$ is a normal subgroup of $M$. Consider the natural homomorphism

$$
\psi: M \rightarrow M /\left(C_{G}\left(L_{1}\right) \times \ldots \times C_{G}\left(L_{k}\right)\right) .
$$

Denoting $\operatorname{Aut}_{G}\left(L_{i}\right)=N_{G}\left(L_{i}\right) / C_{G}\left(L_{i}\right)$ by $A_{i}$ we obtain a homomorphism

$$
\varphi \circ \psi: G \rightarrow\left(A_{1} \times \ldots \times A_{k}\right):(G \rho) \simeq A_{1} \succ(G \rho)=: \bar{G} .
$$

As $C_{G}(T)=\{e\}$, the kernel of $\varphi \circ \psi$ is equal to $C_{G}\left(L_{1}, \ldots, L_{k}\right)=\{e\}$, i. e., $\varphi \circ \psi$ is a monomorphism and we identify $G$ and subgroups of $G$ with their images under $\varphi \circ \psi$. 
Lemma 6. Assume that $G$ possesses a normal subgroup $T$ satisfying conditions (C1), (C2), and (C3). Assume also that $G / T$ is solvable. Consider the monomorphism $\varphi \circ \psi$ defined above. Then the followings hold:

(a) there exists a maximal solvable subgroup $S$ of $G$ such that $G=S T$;

(b) if we choose a maximal solvable subgroup $S$ of $G$ such that $G=S T$, then $\bar{G}$ possesses a maximal solvable subgroup $\bar{S}$ such that $S \leqslant \bar{S}$ and $\bar{G}=\bar{S} T$.

Proof. (a) Consider a minimal subgroup $M$ of $G$ such that $G=M T$. Clearly $M \cap T$ is normal in $M$ and is included in the Frattini subgroup $\Phi(M)$ of $M$. Otherwise $M$ possesses a proper subgroup $M_{1}$ such that $M_{1}(M \cap T)=M$ and so $G=M_{1} T$, a contradiction with the minimality of $M$. Since $\Phi(M)$ is nilpotent and $M /(M \cap T)$ is solvable, it follows that $M$ is solvable. Let $S$ be a maximal solvable subgroup of $G$ containing $M$, then $G=S T$.

(b) Condition (C2) implies $A_{i}=\operatorname{Aut}_{\bar{G}}\left(L_{i}\right)=\operatorname{Aut}_{G}\left(L_{i}\right) \simeq \operatorname{Aut}_{G}\left(L_{1}\right)$ for all $i$. Since $\left[L_{i}, L_{j}\right]=\{e\}$ for $i \neq j$ and $G=S T$, we obtain that

$$
A_{i}=\operatorname{Aut}_{G}\left(L_{i}\right)=N_{G}\left(L_{i}\right) / C_{G}\left(L_{i}\right)=N_{S}\left(L_{i}\right) T / C_{G}\left(L_{i}\right)
$$

and so $A_{i} / L_{i} \simeq N_{S}\left(L_{i}\right) /\left(N_{S}\left(L_{i}\right) \cap L_{i} C_{G}\left(L_{i}\right)\right)$ is solvable. Therefore $\bar{G} /\left(L_{1} \times \ldots \times L_{n}\right) \simeq\left(A_{1} / L_{1}\right)$ ᄀ $(G \rho)$ is solvable. Consider $H=S \cap T$ and denote by $\pi_{i}$ the natural projection $L_{1} \times \ldots \times L_{k} \rightarrow L_{i}$. Put $H_{i}=H^{\pi_{i}}$. Clearly, $H \leqslant H_{1} \times \ldots \times H_{k}$. If $x \in S$ and $L_{i}^{x}=L_{j}$, then $H_{i}^{x}=H_{j}$, since $H$ is normal in $S$. Hence $S$ normalizes $H_{1} \times \ldots \times H_{k}$, and by the maximality of $S$ we have $S \geqslant H_{1} \times \ldots \times H_{k}$, i.e., $H=H_{1} \times \ldots \times H_{k}$. Clearly

$$
N_{T}(H)=N_{L_{1} \times \ldots \times L_{k}}\left(H_{1} \times \ldots \times H_{k}\right)=N_{L_{1}}\left(H_{1}\right) \times \ldots \times N_{L_{k}}\left(H_{k}\right)
$$

By Lemma 5 we have $N_{T}(H)=H$, so $N_{L_{i}}\left(H_{i}\right)=H_{i}$ for $i=1, \ldots, k$. As $N_{S}\left(L_{i}\right) \leqslant N_{A_{i}}\left(H_{i}\right)$, it follows that $A_{i}$ is equal to $N_{A_{i}}\left(H_{i}\right) L_{i}$ and $N_{A_{i}}\left(H_{i}\right)$ is solvable. We obtain that

$$
A_{1} \times \ldots \times A_{k}=\left(N_{A_{1}}\left(H_{1}\right) \times \ldots \times N_{A_{k}}\left(H_{k}\right)\right) T=N_{A_{1} \times \ldots \times A_{k}}(H) T
$$

and $N_{A_{1} \times \ldots \times A_{k}}(H)$ is solvable. Since $\bar{G}=\left(A_{1} \times \ldots \times A_{k}\right) S$, and since $S$ normalizes $H$, it follows that $S$ lies in $N_{\bar{G}}(H)$, and so $\bar{G}=N_{\bar{G}}(H) T$. Moreover $N_{\bar{G}}(H)=N_{A_{1} \times \ldots \times A_{k}}(H)$ is solvable, therefore there exists a maximal solvable subgroup $\bar{S}$ of $\bar{G}$, containing $N_{\bar{G}}(H)$. Thus we obtain that $S \leqslant \bar{S}$ and $\bar{G}=\bar{S} T$.

Let $G$ be a subgroup of $\operatorname{Sym}_{n}$. A partition $P_{1} \sqcup P_{2} \sqcup \ldots \sqcup P_{m}$ of $\{1, \ldots, n\}$ is called an asymmetric partition for $G$, if only the identity element of $G$ fixes the partition, i. e., the equality $P_{j} x=P_{j}$ for all $j=1, \ldots, m$ implies $x=e$. Clearly for every $G$ the partition $P_{1}=\{1\}, P_{2}=\{2\}, \ldots, P_{n}=\{n\}$ is asymmetric.

Lemma 7. [8, Theorem 1.2] Let $G$ be a solvable subgroup of $\mathrm{Sym}_{n}$. Then there exists an asymmetric partition $P_{1} \sqcup P_{2} \sqcup \ldots \sqcup P_{m}=\{1, \ldots, n\}$ with $m \leqslant 5$.

Lemma 8. Let $G$ be a group and let $M$ be a solvable subgroup of $\mathrm{Sym}_{n}$. Assume that there exists $k$ such that for every maximal solvable subgroup $T$ of $G$ the inequalities

$$
\operatorname{Base}_{T}(G) \leqslant k \text { and } \operatorname{Reg}_{T}(G, k)=s \geqslant 5
$$

hold. Then for every maximal solvable subgroup $S$ of $G \geq M$ we have $\operatorname{Base}_{S}(G<M) \leqslant k$. 
Proof. We have $G \succ M=\left(G_{1} \times \ldots \times G_{n}\right): M$. Moreover $S(G \succ M)=S\left(G_{1}\right) \times \ldots \times S\left(G_{n}\right)$, since $C_{M}\left(G_{1} \times \ldots \times G_{n}\right)=\{e\}$. Assume by contradiction that $G>M$ is a counter example to the lemma with $|G<M|$ minimal. Then clearly $S(G<M)=\{e\}$, i.e., $S(G)=\{e\}$, otherwise we substitute $G$ by $G / S(G)$ and proceed by induction.

Since $G>M$ is a counter example to the lemma, there exists a maximal solvable subgroup $S$ of $G>M$ such that for every $x_{1}, \ldots, x_{k} \in G<M$ we have $S^{x_{1}} \cap \ldots \cap S^{x_{k}} \neq\{e\}$. It is clear that $\left(G_{1} \times \ldots \times G_{n}\right) S=G$ ? $M$, otherwise consider the image $\bar{S}$ of $S$ under the natural homomorphism $G \prec M \rightarrow M$. We obtain that $\left(G_{1} \times \ldots \times G_{n}\right) S=G \imath \bar{S}<G \imath M$, so we substitute $G \imath M$ by $G \succ \bar{S}$ and proceed by induction. The minimality of $G>M$ implies also that $M$ is transitive, otherwise we would obtain that $G \prec M \leqslant\left(G \prec M_{1}\right) \times\left(G \prec M_{2}\right)$, where $M_{1} \leqslant \mathrm{Sym}_{m}, M_{2} \leqslant \mathrm{Sym}_{n-m}$, and proceed by induction. Indeed denote the projections of $G<M$ onto $G<M_{1}$ and $G<M_{2}$ by $\pi_{1}$ and $\pi_{2}$ respectively. Up to renumbering we may suppose that $G \imath M_{1}=\left(G_{1} \times \ldots \times G_{m}\right): M_{1}$ and $G \imath M_{2}=\left(G_{m+1} \times \ldots \times G_{n}\right): M_{2}$. Denote $G_{1} \times \ldots \times G_{m}$ by $E_{1}$ and $G_{m+1} \times \ldots \times G_{n}$ by $E_{2}$. Since $G \imath M=\left(G_{1} \times \ldots \times G_{n}\right) S, E_{1} \leqslant \operatorname{Ker}\left(\pi_{2}\right)$, and $E_{2} \leqslant \operatorname{Ker}\left(\pi_{1}\right)$, it follows that $(G \succ M) \pi_{i}=E_{i}\left(S \pi_{i}\right)$ (we identify $E_{i} \pi_{i}$ with $E_{i}$, since $\left.E_{i} \pi_{i} \simeq E_{i}\right)$. By induction for each $i \in\{1,2\}$ there exist elements $x_{1, i}, \ldots, x_{k, i}$ of $E_{i}\left(S \pi_{i}\right)$ such that

$$
\left(S \pi_{i}\right)^{x_{1, i}} \cap \ldots \cap\left(S \pi_{i}\right)^{x_{k, i}}=\{e\}
$$

Since $G \pi_{i}=E_{i}\left(S \pi_{i}\right)$, we may assume that $x_{1, i}, \ldots, x_{k, i}$ are in $E_{i}$. Consider $x_{1}=x_{1,1} x_{1,2}, \ldots, x_{k}=$ $x_{k, 1} x_{k, 2}$. Since (2) is true for every $i$, we have

$$
S^{x_{1}} \cap \ldots \cap S^{x_{k}}=\{e\},
$$

and $G$ is not a counter example.

Consider $L=S \cap\left(G_{1} \times \ldots \times G_{n}\right)$ and denote by $\pi_{i}$ the natural projection $G_{1} \times \ldots \times G_{n} \rightarrow G_{i}$. Put $L_{i}=L^{\pi_{i}}$. Clearly $L \leqslant L_{1} \times \ldots \times L_{n}$. If $x \in S$ and $G_{i}^{x}=G_{j}$, then $L_{i}^{x}=L_{j}$, since $L$ is normal in $S$. Hence $S$ normalizes $L_{1} \times \ldots \times L_{n}$ and by the maximality of $S$ we have $L=L_{1} \times \ldots \times L_{n}$.

Clearly $N_{G_{1} \times \ldots \times G_{n}}\left(L_{1} \times \ldots \times L_{n}\right)=N_{G_{1}}\left(L_{1}\right) \times \ldots \times N_{G_{n}}\left(L_{n}\right)$. By Lemma 5 we obtain that $N_{G_{1} \times \ldots \times G_{n}}\left(L_{1} \times\right.$ $\left.\ldots \times L_{n}\right)=L_{1} \times \ldots \times L_{n}$, hence $N_{G_{i}}\left(L_{i}\right)=L_{i}$ for $i=1, \ldots, n$. Denote by $\Omega_{i}$ the set $\left\{L_{i}^{x} \mid x \in G_{i}\right\}$, then $G_{i}$ acts on $\Omega_{i}$ by conjugation. Since $N_{G_{i}}\left(L_{i}\right)=L_{i}$, it follows that $L_{i}$ is the point stabilizer under this action. Set $\Omega=\Omega_{1} \times \ldots \times \Omega_{n}$. For every $x \in G \imath M$ and for every $i$ we have $L_{i}^{x} \leqslant G_{j}$ for some $j$. We show that

$$
\text { if } L_{i}^{x} \leqslant G_{j} \text { then } L_{i}^{x} \in L_{j}^{G_{j}} \text {, i.e., there exists } y \in G_{j} \text { such that } L_{j}^{y}=L_{i}^{x} \text {. }
$$

Since $\left(G_{1} \times \ldots \times G_{n}\right): M=\left(G_{1} \times \ldots \times G_{n}\right) S$, it follows that there exists $s \in S$ with $G_{i}^{s}=G_{j}$. We also have $L_{i}^{s}=L_{j}$, since $L$ is normal in $S$. Thus $L_{i}^{x}=L_{j}^{s^{-1} x}$. Now $s^{-1} x=g_{1} \cdot \ldots \cdot g_{n} \cdot h$, where $g_{i} \in G_{i}$ for $i=1, \ldots, n$ and $h \in M$. Since $M$ permutes the $G_{i}$-s, it follows that for every $i=1, \ldots, n$, either $G_{i}^{h} \cap G_{i}=\{e\}$, or $h$ centralizes $G_{i}$. Thus we obtain that $L_{j}^{s^{-1} x}=L_{j}^{g_{j}}$. So $G>M$ acts by conjugation on $\Omega$ and $S$ is the stabilizer of the point $\left(L_{1}, \ldots, L_{n}\right)$. Therefore we need to show that $\Omega^{k}$ possesses a $(G<M)$-regular orbit.

The conditions of the lemma imply that there exist $G_{1}$-regular points $\omega_{1}, \ldots, \omega_{s} \in \Omega_{1}^{k}$ lying in distinct $G_{1}$-orbits. If we choose $h_{1}=e, h_{2}, \ldots, h_{n} \in M$ so that $G_{1}^{h_{i}}=G_{i}$, then $\omega_{1}^{h_{i}}, \ldots, \omega_{s}^{h_{i}} \in \Omega_{i}^{k}$ are $G_{i}$-regular points, and (3) implies that they are in distinct $G_{i}$-orbits. We set $\omega_{i, j}=\omega_{i}^{h_{j}}$. By Lemma 7 there exists an asymmetric partition $P_{1} \sqcup P_{2} \sqcup P_{3} \sqcup P_{4} \sqcup P_{5}=\{1, \ldots, n\}$ for $M$. Since $s \geqslant 5$ we can choose $\omega=\left(\omega_{i_{1}, 1}, \ldots, \omega_{i_{n}, n}\right)$ so that $i_{t}=i_{j}$ if and only if $t, j$ lie in the same $P_{l}$. Now we show that $\omega \in \Omega^{k}$ is a $(G \succ M)$-regular point. Indeed, consider $g=\left(g_{1} \ldots g_{n}\right) h$, where $g_{i} \in G_{i}$ for $i=1, \ldots, n$ and $h \in M$, and assume that $\omega g=\omega$. It follows that $\omega h^{-1}=\omega\left(g_{1} \ldots g_{n}\right)$, i.e.,

$$
\left(\omega_{i_{1}, 1}, \ldots, \omega_{i_{n}, n}\right) h^{-1}=\left(\omega_{\left.i_{1} h\right)}, 1, \ldots, \omega_{i_{(n h)}, n}\right)=\left(\omega_{i_{1}, 1} g_{1}, \ldots, \omega_{i_{n}, n} g_{n}\right)
$$


Therefore $\omega_{i_{(j h), j}}$ and $\omega_{i_{j}, j}$ are in the same $G_{j}$-orbit, i.e., $i_{(j h)}=i_{j}$. By construction, $j h$ and $j$ are in the same $P_{l}$. Whence $h$ stabilizes the partition $P_{1} \sqcup P_{2} \sqcup P_{3} \sqcup P_{4} \sqcup P_{5}$ and $h=e$. We obtain that $\left(\omega_{i_{1}, 1}, \ldots, \omega_{i_{n}, n}\right)=\left(\omega_{i_{1}, 1} g_{1}, \ldots, \omega_{i_{n}, n} g_{n}\right)$. By construction, $\omega_{i_{j}, j}$ is a $G_{j}$-regular point for every $j=1, \ldots, n$, so $g_{1}=\ldots=g_{n}=e$, i.e., $g=e$ and $\omega \in \Omega^{k}$ is a $(G<M)$-regular point.

\section{Proof of the main theorem and the corollary}

Proof of Theorem 1 Assume that the claim is false and $G$ is a counter example of minimal order. Fix a maximal solvable subgroup $S$ of $G$ with $\operatorname{Base}_{S}(G)>k$.

Assume that $S(G) \neq\{e\}$. Then there exists a minimal elementary abelian normal subgroup $K$ of $G$. Since elements from distinct minimal normal subgroups commute, we may suppose that $G_{1} \leqslant K$ and there exists $l$ such that $G_{l}=K$, i.e., the composition series (1) is a refinement of a chief series starting with $K$. In this case, if

$$
-G \rightarrow G / K=\bar{G}
$$

is the natural homomorphism, then

$$
\{\bar{e}\}=\bar{G}_{l}<\bar{G}_{l+1}<\ldots<\bar{G}_{n}=\bar{G}
$$

is a composition series of $\bar{G}$ that is a refinement of a chief series of $\bar{G}$. Moreover, for every nonabelian $\bar{G}_{i} / \bar{G}_{i-1}$, Lemma 4 implies $\operatorname{Aut}_{\bar{G}}\left(\bar{G}_{i} / \bar{G}_{i-1}\right) \simeq \operatorname{Aut}_{G}\left(G_{i} / G_{i-1}\right)$. Since $G$ satisfies (Orb-solv) for some $k$, we obtain that $\bar{G}$ satisfies (Orb-solv) for the same $k$. In view of the minimality of $G$, there exist $x_{1}, \ldots, x_{k} \in G$ such that

$$
\bar{S}^{\bar{x}_{1}} \cap \ldots \cap \bar{S}^{\bar{x}_{k}}=S(\bar{G}) .
$$

Now $K \leqslant S(G)$, hence $\overline{S(G)}=S(\bar{G})$. Therefore $S^{x_{1}} \cap \ldots \cap S^{x_{k}}=S(G)$, i.e., $G$ is not a counter example.

Thus we may assume that $S(G)=\{e\}$. Consider the generalized Fitting subgroup $F^{*}(G)$ of $G$. Since $S(G)=\{e\}$, we obtain that $F^{*}(G)=L_{1} \times \ldots \times L_{n}$ is a product of nonabelian simple groups and, by [7, Theorem 9.8], $C_{G}\left(F^{*}(G)\right)=Z\left(F^{*}(G)\right)=\{e\}$. In particular, $S\left(F^{*}(G) S\right)=\{e\}$. If $F^{*}(G) S \neq G$, then, in view of the minimality of $G$, there exist $x_{1}, \ldots, x_{k} \in F^{*}(G) S$ such that $S^{x_{1}} \cap \ldots \cap S^{x_{k}}=$ $S\left(F^{*}(G) S\right)=\{e\}$, i.e., $G$ is not a counter example. It follows that $G=F^{*}(G) S$. Moreover, since $L_{1}, \ldots, L_{n}$ are nonabelian simple, [6, Satz 12.5, p. 69] implies that $G$, acting by conjugation, permutes the elements of $\left\{L_{1}, \ldots, L_{n}\right\}$.

Set $E_{1}:=\left\langle L_{1}^{S}\right\rangle$ and $E_{2}=\left\langle L_{i} \mid L_{i} \notin\left\{L_{1}^{s} \mid s \in S\right\}\right\rangle$. Since $F^{*}(G)=L_{1} \times \ldots \times L_{n}$, we obtain that $F^{*}(G)=E_{1} \times E_{2}$, where $E_{1}$ and $E_{2}$ are $S$-invariant subgroups. By [6, Hilfssatz 9.6, p. 48] there exists a homomorphism $G \rightarrow G / C_{G}\left(E_{1}\right) \times G / C_{G}\left(E_{2}\right)$, such that the image of $G$ is a subdirect product of $G / C_{G}\left(E_{1}\right)$ and $G / C_{G}\left(E_{2}\right)$, while the kernel is equal to $C_{G}\left(E_{1}\right) \cap C_{G}\left(E_{2}\right)=C_{G}\left(F^{*}(G)\right)=\{e\}$. Denote the projections of $G$ onto $G / C_{G}\left(E_{1}\right)$ and $G / C_{G}\left(E_{2}\right)$ by $\pi_{1}$ and $\pi_{2}$ respectively. Since $G=F^{*}(G) S$, $E_{1} \leqslant \operatorname{Ker}\left(\pi_{2}\right)$ and $E_{2} \leqslant \operatorname{Ker}\left(\pi_{1}\right)$, it follows that $G \pi_{1}=E_{1}\left(S \pi_{1}\right)$ and $G \pi_{2}=E_{2}\left(S \pi_{2}\right)$ (we identify $E_{i} \pi_{i}$ with $E_{i}$ since $E_{i} \pi_{i} \simeq E_{i}$ ).

Suppose that $E_{1} \neq F^{*}(G)$. Then, by induction for each $i \in\{1,2\}$ there exist elements $x_{1, i}, \ldots, x_{k, i}$ of $E_{i}\left(S \pi_{i}\right)$ such that

$$
\left(S \pi_{i}\right)^{x_{1, i}} \cap \ldots \cap\left(S \pi_{i}\right)^{x_{k, i}}=\{e\} .
$$

Since $G \pi_{i}=E_{i}\left(S \pi_{i}\right)$, we may assume that $x_{1, i}, \ldots, x_{k, i}$ are in $E_{i}$. Consider $x_{1}=x_{1,1} x_{1,2}, \ldots, x_{k}=$ $x_{k, 1} x_{k, 2}$. Since (4) is true for every $i$ and $\operatorname{Ker}\left(\pi_{1}\right) \cap \operatorname{Ker}\left(\pi_{2}\right)=\{e\}$, we have

$$
S^{x_{1}} \cap \ldots \cap S^{x_{k}}=\{e\}
$$


and $G$ is not a counter example.

Therefore $E_{1}=F^{*}(G)$ and $S$ acts transitively on $\left\{L_{1}, \ldots, L_{n}\right\}$. Since $\operatorname{Aut}_{G}\left(L_{1}\right)$ satisfies (Orb-solv) for some $k$, we may assume that $n>1$. By Lemma 6 and by the discussion preceding it, we may assume that $G=\left(A_{1} \times \ldots \times A_{n}\right): K=A_{1} \succ K$, where $A_{i}=\operatorname{Aut}_{G}\left(L_{i}\right), K=G \rho \leqslant \operatorname{Sym}_{n}$ and $\rho$ is the permutation representation of $G$ on the set $\left\{L_{1}, \ldots, L_{n}\right\}$. Since $G=F^{*}(G) S$, we see that $K=S \rho$ is solvable. Lemma 8 (applied with $G=A$ ) implies that $\operatorname{Base}_{S}(G) \leqslant k$ for every maximal solvable subgroup $S$ of $G$. This final contradiction completes the proof.

Proof of Corollary 2 Let $G$ be a group satisfying (Orb-solv) for $k=5$. Assume that $G$ possesses a solvable $\pi$-Hall subgroup $H$. Consider the natural homomorphism

$$
-: G \rightarrow G / S(G)
$$

Since $H$ is solvable, it follows that there exists a maximal solvable subgroup $S$ of $G$ with $H \leqslant S$. By Theorem 1 there exist $x_{1}, x_{2}, x_{3}, x_{4}, x_{5}$ such that

$$
S^{x_{1}} \cap S^{x_{2}} \cap S^{x_{3}} \cap S^{x_{4}} \cap S^{x_{5}}=S(G) .
$$

Thence $H^{x_{1}} \cap H^{x_{2}} \cap H^{x_{3}} \cap H^{x_{4}} \cap H^{x_{5}} \leqslant S(G)$ and $\bar{H}^{\bar{x}_{1}} \cap \bar{H}^{\bar{x}_{2}} \cap \bar{H}^{\bar{x}_{3}} \cap \bar{H}^{\bar{x}_{4}} \cap \bar{H}^{\bar{x}_{5}}=\{\bar{e}\}$. Consider $H \cap S(G)=K$. As $S(G)$ is normal in $G$, we obtain that $K$ is a $\pi$-Hall subgroup of $S(G)$. In view of [4, Theorem 1.3] or [10, Theorem 1.3] there exist $x, y \in S(G)$ such that $K \cap K^{x} \cap K^{y}=O_{\pi}(S(G))$. As $O_{\pi}(G)$ is a normal $\pi$-subgroup of $G$ and $H$ is a solvable $\pi$-Hall subgroup, we get $O_{\pi}(G) \leqslant H$ and $O_{\pi}(G)$ is solvable. Therefore $O_{\pi}(G) \leqslant S(G)$ and $O_{\pi}(G) \leqslant O_{\pi}(S)$. Thus $O_{\pi}(G)=O_{\pi}(S)$. Therefore there exist $y_{1}, y_{2}, y_{3}, y_{4}, y_{5}$ such that $K^{y_{1}} \cap K^{y_{2}} \cap K^{y_{3}} \cap K^{y_{4}} \cap K^{y_{5}}=O_{\pi}(G)$. Denote by $M_{i}$ the complete preimage of $\bar{H}^{\bar{x}_{i}}$ in $G$, for $i=1,2,3,4,5$. Since $K^{y_{i}}$ and $S(G) \cap H^{x_{i}}$ are $\pi$-Hall subgroup of $S(G)$ and since $S(G)$ is solvable, there exists $z_{i} \in S(G)$ with

$$
K^{y_{i}}=\left(S(G) \cap H^{x_{i}}\right)^{z_{i}}=S(G) \cap H^{x_{i} z_{i}} .
$$

Clearly $\bar{H}^{\bar{x}_{i}}=\bar{H}^{\bar{x}_{i} \bar{z}_{i}}$ and so

$$
H^{x_{1} z_{1}} \cap \ldots \cap H^{x_{5} z_{5}} \subseteq S(G)
$$

Hence $H^{x_{1} z_{1}} \cap \ldots \cap H^{x_{5} z_{5}}=K^{y_{1}} \cap \ldots \cap K^{y_{5}}=O_{\pi}(G)$.

\section{Final notes}

In this final section we consider two natural problems related with the main subject of the paper.

Problem 2. Given $H \leqslant G$, how to find a lower bound for $\operatorname{Base}_{H}(G)$ ?

Problem 3. Is it possible to remove condition $\operatorname{Reg}_{S}\left(\operatorname{Aut}_{G}\left(G_{i}, G_{i-1}\right), k\right) \geqslant 5$ ?

Consider Problem 2 first. Assume that $G$ acts faithfully and transitively on $\Omega$, and $\operatorname{Base}(G)=k>1$. Consider a $G$-regular point $\left(\omega_{1}, \ldots, \omega_{k}\right) \in \Omega^{k}$. Clearly $\omega_{i} \neq \omega_{j}$ for $i \neq j$. Hence we obtain

$$
|G|=\left|\left(\omega_{1}, \ldots, \omega_{k}\right) G\right| \leqslant|\Omega| \cdot(|\Omega|-1) \cdot \ldots \cdot(|\Omega|-k+1)<|\Omega|^{k} .
$$

Now consider $H \leqslant G$ such that $H$ is not normal in $G$ and assume that $\operatorname{Base}_{H}(G)=k$. Inequality (5) implies $\left|G / H_{G}\right|<|G: H|^{k}$, and so

$$
\left|H / H_{G}\right|<|G: H|^{k-1} \text {. }
$$


Inequality (6) gives us the lower bound for $\operatorname{Base}_{H}(G)$. Namely,

$$
\operatorname{Base}_{H}(G) \geqslant \min \left\{k|| G:\left.H\right|^{k-1}>\left|H / H_{G}\right|\right\} .
$$

Theorem 2.13 from [1] implies that there exists a constant $c$ such that every finite group possessing a solvable subgroup of index $n$ possesses a normal solvable subgroup of index at most $n^{c}$. Conjecture 6.6 from the same paper asserts that $c \leqslant 7$. Therefore (6) implies that part (a) of Problem 17.41 from the "Kourovka notebook" is a strengthen of the original Conjecture 6.6 from [1].

Now we discuss Problem 3. First we show that the condition $\operatorname{Reg}_{S}\left(\operatorname{Aut}_{G}\left(G_{i}, G_{i-1}\right), k\right) \geqslant 5$ is essential. The following example is given by V.I.Zenkov in [12].

Example 9. Consider $G=\operatorname{Sym}_{5} \prec \operatorname{Sym}_{2}$ and $S=\operatorname{Sym}_{4} \imath \operatorname{Sym}_{2}$. It is evident that $\mathrm{Alt}_{5}$ is the unique nonabelian composition factor of $G$ (however there are two nonabelian composition factors isomorphic to $\left.\mathrm{Alt}_{5}\right)$. It is also easy to see, that for every solvable subgroup $T$ of $\operatorname{Sym}_{5}=\operatorname{Aut}\left(\mathrm{Alt}_{5}\right)$ we have $\operatorname{Base}_{T}\left(\operatorname{Sym}_{5}\right) \leqslant 4$. In this case we have $\operatorname{Reg}_{\text {Sym }_{4}}\left(\operatorname{Sym}_{5}, 4\right)=1$ and the lemma from [12] implies that $\operatorname{Base}_{S}(G)=5$.

The next example obtained in [11] shows that there exists an almost simple group $G$ possessing a solvable subgroup $S$ with $\operatorname{Base}_{S}(G)=5$.

Example 10. Consider $G=\operatorname{Sym}_{8}$ and $S=\operatorname{Sym}_{4} \imath \operatorname{Sym}_{2}$. Then $\operatorname{Base}_{S}(G)=5$. Notice also that in [11] the inequality $\operatorname{Reg}_{S}(G, 5) \geqslant 12$ is proven. Furthermore $|S|<|G: S|^{2}$ and so in this case $\operatorname{Base}_{S}(G)$ is greater than the lower bound given by (7).

We show that if $k \geqslant 6$, then we can guarantee that $\operatorname{Reg}_{S}\left(\operatorname{Aut}_{G}\left(G_{i} / G_{i-1}\right), k\right) \geqslant 5$. More precisely, the following lemma holds.

Lemma 11. Let $G$ be a transitive permutation group acting on $\Omega=\{1, \ldots, n\}$ and let the stabilizer $S$ of 1 be solvable. Assume that $k=\max \{\operatorname{Base}(G), 6\}$. Then $\operatorname{Reg}(G, k) \geqslant 5$.

We start with a technical result.

Lemma 12. Let $G$ be a transitive subgroup of $\operatorname{Sym}_{n}$. Denote $\Omega=\{1, \ldots, n\}$. Let $H$ be the stabilizer of 1 in $G$.

(a) $\left(1, i_{2}, \ldots, i_{k}\right)$ and $\left(1, j_{2}, \ldots, j_{k}\right)$ are in the same $G$-orbit if and only if $\left(i_{2}, \ldots, i_{k}\right)$ and $\left(j_{2}, \ldots, j_{k}\right)$ are in the same H-orbit;

(b) every $G$-orbit of $\Omega^{k}$ contains an element $\left(1, i_{2}, \ldots, i_{k}\right)$;

(c) $\left(1, i_{2}, \ldots, i_{k}\right)$ is a $G$-regular point if and only if $\left(i_{2}, \ldots, i_{k}\right)$ is an H-regular point;

(d) the number of $G$-orbits in $\Omega^{k}$ is equal to the number of $H$-orbits in $(\Omega \backslash\{1\})^{k-1}$;

Proof. (a) Evident.

(b) Follows from the fact that $G$ is transitive.

(c) If $\left(1, i_{2}, \ldots, i_{k}\right)$ is a $G$-regular point, then $\left(1, i_{2}, \ldots, i_{k}\right) g=\left(1, i_{2}, \ldots, i_{k}\right)$ implies $g=e$. Assume that $h \in H$ is chosen so that $\left(i_{2}, \ldots, i_{k}\right) h=\left(i_{2}, \ldots, i_{k}\right)$. Since $H$ is the stabilizer of 1 , it follows that $\left(1, i_{2}, \ldots, i_{k}\right) h=\left(1, i_{2}, \ldots, i_{k}\right)$, hence $h=e$ and $\left(i_{2}, \ldots, i_{k}\right)$ is an $H$-regular point. Conversely, if $\left(i_{2}, \ldots, i_{k}\right)$ is an $H$-regular point and $\left(1, i_{2}, \ldots, i_{k}\right) g=\left(1, i_{2}, \ldots, i_{k}\right)$, we obtain $g \in H$, and $\left(i_{2}, \ldots, i_{k}\right) g=$ $\left(i_{2}, \ldots, i_{k}\right)$, hence $g=e$ and $\left(1, i_{2}, \ldots, i_{k}\right)$ is a $G$-regular point.

(d) Clear from (a), (b) and (c). 
Proof of Lemma 11. In view of Lemma 12, we have that $S$ acts on $\Theta=\Omega \backslash\{1\}$ and the number of $G$-regular orbits on $\Omega^{k}$ is equal to the number of $S$-regular orbits on $\Theta^{k-1}$. Thus we need to prove that $\operatorname{Reg}(S, k-1) \geqslant 5$, where $S$ acts on $\Theta$. Since $k \geqslant \operatorname{Base}(G)$, Lemma 12 (c) implies that there exist $\theta_{1}, \ldots, \theta_{k-1} \in \Theta$ such that $\left(\theta_{1}, \ldots, \theta_{k-1}\right)$ is an $S$-regular point in $\Theta^{k-1}$.

Consider $\Delta=\left\{\theta_{1}, \ldots, \theta_{k-1}\right\}$, let $T$ be the setwise stabilizer of $\Delta$ in $S$, i.e., $T=\{x \in S \mid \Delta x=\Delta\}$. It is clear that $\left(\theta_{1 \sigma}, \ldots, \theta_{(k-1) \sigma}\right)$ is an $S$-regular point for every $\sigma \in \operatorname{Sym}_{k-1}$. Moreover if $\sigma, \tau \in \operatorname{Sym}_{k-1}$, then $\left(\theta_{1 \sigma}, \ldots, \theta_{(k-1) \sigma}\right)$ and $\left(\theta_{1 \tau}, \ldots, \theta_{(k-1) \tau}\right)$ are in the same $S$-orbit if and only if there exists $x \in T$ such that $\left(\theta_{1 \sigma}, \ldots, \theta_{(k-1) \sigma}\right)^{x}=\left(\theta_{1 \tau}, \ldots, \theta_{(k-1) \tau}\right)$. Consider the restriction homomorphism $\varphi: T \rightarrow \operatorname{Sym}(\Delta)$. Since $\left(\theta_{1}, \ldots, \theta_{k-1}\right)$ is an $S$-regular point (and so a $T$-regular point), it follows that $\operatorname{Ker}(\varphi)=\{e\}$, i.e., $\varphi$ is injective.

Assume that $k \geqslant 9$ first. Consider an asymmetric partition $P_{1} \sqcup P_{2} \sqcup P_{3} \sqcup P_{4} \sqcup P_{5}=\left\{\theta_{1}, \theta_{2}, \ldots, \theta_{k-1}\right\}$ for $T^{\varphi}$ (the existence of the partition follows by Lemma 7). Without loss of generality we may assume that $\left|P_{1}\right| \geqslant\left|P_{2}\right| \geqslant\left|P_{3}\right| \geqslant\left|P_{4}\right| \geqslant\left|P_{5}\right|$. Since $k \geqslant 9$ (and so $\left|\left\{\theta_{1}, \theta_{2}, \ldots, \theta_{k-1}\right\}\right| \geqslant 8$ ) it follows that either $\left|P_{1}\right| \geqslant 3$, or $\left|P_{1}\right|=\left|P_{2}\right|=\left|P_{3}\right|=2$.

If $\left|P_{1}\right| \geqslant 3$, then, up to renumbering, we may assume that $\theta_{1}, \theta_{2}, \theta_{3} \in P_{1}$. In this case for every distinct $\sigma, \tau \in \mathrm{Sym}_{3}$ we have that $\left(\theta_{1 \sigma}, \theta_{2 \sigma}, \theta_{3 \sigma}, \theta_{4} \ldots, \theta_{k-1}\right)$ and $\left(\theta_{1 \tau}, \theta_{2 \tau}, \theta_{3 \tau}, \theta_{4}, \ldots, \theta_{k-1}\right)$ are in distinct $T^{\varphi}$-orbits, thus these points are in distinct $T$-orbits, and so in distinct $S$-orbits. $\operatorname{So} \operatorname{Reg}(S, k-1) \geqslant$ $\left|\mathrm{Sym}_{3}\right|=6$ in this case.

If $\left|P_{1}\right|=\left|P_{2}\right|=\left|P_{3}\right|=2$, then, up to renumbering, we may assume that $\theta_{1}, \theta_{2} \in P_{1}, \theta_{3}, \theta_{4} \in P_{2}$, and $\theta_{5}, \theta_{6} \in P_{3}$. In this case for every distinct $\sigma, \tau \in \operatorname{Sym}(\{1,2\}) \times \operatorname{Sym}(\{3,4\}) \times \operatorname{Sym}(\{5,6\})$ we have that

$$
\left(\theta_{1 \sigma}, \theta_{2 \sigma}, \theta_{3 \sigma}, \theta_{4 \sigma}, \theta_{5 \sigma}, \theta_{6 \sigma}, \theta_{7} \ldots, \theta_{k-1}\right) \text { and }\left(\theta_{1 \tau}, \theta_{2 \tau}, \theta_{3 \tau}, \theta_{4 \tau}, \theta_{5 \tau}, \theta_{6 \tau}, \theta_{7} \ldots, \theta_{k-1}\right)
$$

are in distinct $T^{\varphi}$-orbits, thus these points are in distinct $T$-orbits, and so in distinct $S$-orbits. So $\operatorname{Reg}(S, k-1) \geqslant|\operatorname{Sym}(\{1,2\}) \times \operatorname{Sym}(\{3,4\}) \times \operatorname{Sym}(\{5,6\})|=8$ in this case.

Now assume that $6 \leqslant k \leqslant 8$. Denote by $\Xi$ the subset $\left\{\left(\theta_{1 \sigma}, \ldots, \theta_{(k-1) \sigma}\right) \mid \sigma \in \operatorname{Sym}_{k-1}\right\}$ of $\Delta^{k-1}$. Then $T^{\varphi}$ acts on $\Xi$ and every point of $\Xi$ is $T^{\varphi}$-regular. Moreover $|\Xi|=\left|\operatorname{Sym}_{k-1}\right|=(k-1)$ !. We also have that $T^{\varphi}$ is a solvable subgroup of $\mathrm{Sym}_{k-1}$. It is immediate (from [3], for example), that $\left|T^{\varphi}\right| \leqslant 24$ for $k=6,\left|T^{\varphi}\right| \leqslant 72$ for $k=7$, and $\left|T^{\varphi}\right| \leqslant 144$ for $k=8$. Now the number of $T^{\varphi}$-orbits on $\Xi$ is equal to $\frac{(k-1) !}{\left|T^{\varphi}\right|}$ and direct computations show that this number is at least 5 .

At the end of the paper we show, how $\operatorname{Reg}_{S}(G, k)$ can be applied for the computational purposes. If we have a group $G$ and a maximal solvable subgroup $S$ of $G$, then Theorem 1 gives us an idea, how to find $\operatorname{Base}_{S}(G)$, or, at least, how to find an upper bound for $\operatorname{Base}_{S}(G)$. However, for computation purposes it is also important to find the base of $G$ with respect to $S$, i.e., elements $x_{1}, \ldots, x_{k}$ such that $S^{x_{1}} \cap \ldots \cap S^{x_{k}}=S_{G}$. In general it is computationally very hard to find these elements and we can suggest just a probabilistic approach in this direction. Denote by $\Omega$ the set of right cosets of $S$ in $G$. If one knows that $\operatorname{Reg}_{S}(G, k) \geqslant s$ and $|G: S|=|\Omega|=n$, then $\left|\Omega^{k}\right|=n^{k}$, while $\Omega^{k}$ possesses at least $s\left|G / S_{G}\right|$ regular points. So the probability that $k$ randomly chosen elements from $\Omega$ form a base of $G$ with respect to $S$ is not less than

$$
\varepsilon=\frac{s \cdot\left|G / S_{G}\right|}{n^{k}} \geqslant \frac{s}{n^{k-1}} .
$$

The final lemma allows to obtain a lower bound for $\operatorname{Reg}_{S}(G, k)$ in a particular case.

Lemma 13. Let $G$ be a group and let $M$ be a solvable subgroup of $\mathrm{Sym}_{n}$. Assume that there exists $k$ such that for every maximal solvable subgroup $T$ of $G$ the inequalities

$$
\operatorname{Base}_{T}(G) \leqslant k \text { and } \operatorname{Reg}_{T}(G, k)=s \geqslant 5
$$

hold. Then for every maximal solvable subgroup $S$ of $G>M$ we have $\operatorname{Reg}_{S}(G \succ M, k) \geqslant s$. 
Proof. In the proof we preserve the notation from the proof of Lemma 8. Assume that the claim is false and $G \prec M$ is a counter example with $|G \imath M|$ minimal. Then $G \prec M$ possesses a maximal solvable subgroup $S$ with $\operatorname{Reg}_{S}(G<M, k)<s$. The minimality of $|G<M|$ implies that $S(G)=\{e\}$ (and so $S(G \succ M)=\{e\})$, and $G \succ M=\left(G_{1} \times \ldots \times G_{n}\right) S$. Since $G \succ M$ is a minimal counter example we also obtain that $M$ is transitive. Indeed, assume that $M$ is not transitive, so $G \imath M \leqslant\left(G \succ M_{1}\right) \times\left(G \nmid M_{2}\right)$, where $M_{1} \leqslant \operatorname{Sym}_{m}$ and $M_{2} \leqslant \operatorname{Sym}_{n-m}$. Up to renumbering we may suppose that $G \imath M_{1}=\left(G_{1} \times \ldots \times G_{m}\right): M_{1}$ and $G \imath M_{2}=\left(G_{m+1} \times \ldots \times G_{n}\right): M_{2}$. Denote $G_{1} \times \ldots \times G_{m}$ by $E_{1}$ and $G_{m+1} \times \ldots \times G_{n}$ by $E_{2}$. Consider the projections $\pi_{1}$ and $\pi_{2}$ of $G \imath M$ onto $G \imath M_{1}$ and $G \imath M_{2}$ respectively. Since $G \imath M=\left(G_{1} \times \ldots \times G_{n}\right) S$, $E_{1} \leqslant \operatorname{Ker}\left(\pi_{2}\right)$, and $E_{2} \leqslant \operatorname{Ker}\left(\pi_{1}\right)$, it follows that $(G<M) \pi_{i}=E_{i}\left(S \pi_{i}\right)$ (we identify $E_{i} \pi_{i}$ with $E_{i}$ since $\left.E_{i} \pi_{i} \simeq E_{i}\right)$. By induction for each $i \in\{1,2\}$ there exists at least $s(G<M)$-regular orbits with representatives

$$
\left(S x_{1, i, 1}, \ldots, S x_{k, i, 1}\right), \ldots,\left(S x_{1, i, s}, \ldots, S x_{k, i, s}\right) \text {. }
$$

As we noted in the proof of Lemma 8, we may assume that $x_{l, i, j} \in E_{i}$ for $l=1, \ldots, k, i=1,2$, $j=1, \ldots, s$. If we denote $x_{l, 1, j} \cdot x_{l, 2, j}$ by $x_{l, j}$, then for each $j=1, \ldots, s$ we obtain that $\left(S x_{1, j}, \ldots, S x_{k, j}\right)$ is an $(G \backslash M)$-regular point. Clearly, $\left(S x_{1, j}, \ldots, S x_{k, j}\right)$ and $\left(S x_{1, l}, \ldots, S x_{k, l}\right)$ are in distinct $(G \succ M)$-orbits for $j \neq l$.

Thus $M$ is transitive and $G>M=\left(G_{1} \times \ldots \times G_{n}\right) S$. Recall that symbols $L_{1}, \ldots, L_{n}, \Omega_{1}, \ldots, \Omega_{n}$, $\Omega, \omega_{i, j}$ for $i=1, \ldots, s, j=1, \ldots, n, P_{1}, P_{2}, P_{3}, P_{4}, P_{5}$ are defined in the proof of Lemma 8 . In the proof of Lemma 8 we have shown that a point $\omega=\left(\omega_{i_{1}, 1}, \ldots, \omega_{i_{n}, n}\right)$ chosen so that $i_{t}=i_{j}$ if and only if $t, j$ are in the same $P_{l}$ is an $(G \succ M)$-regular point. If $s>5$, then for each $i=1, \ldots, s$ we can choose $\omega^{i}=\left(\omega_{i_{1}, 1}, \ldots, \omega_{i_{n}, n}\right)$ so that $i_{t}=i_{j}$ if and only if $t, j$ are in the same $P_{l}$ and $i \notin\left\{i_{1}, \ldots, i_{n}\right\}$. Now (3) implies that $\omega^{1}, \ldots, \omega^{s}$ are in distinct $(G>M)$-orbits, so $G \succ M$ is not a counter example. Thus $s=5$.

Consider $\omega=\left(\omega_{i_{1}, 1}, \ldots, \omega_{i_{n}, n}\right)$ and $\theta=\left(\omega_{j_{1}, 1}, \ldots, \omega_{j_{n}, n}\right)$, and assume that $\omega$ and $\theta$ are in the same $(G\urcorner M)$-orbit. Therefore there exists $g=g_{1} \ldots g_{n} h$, where $g_{i} \in G_{i}$ and $h \in M$, such that $\omega g=\theta$. The equality $\omega g=\theta$ can be written as

$$
\left(\omega_{i_{1}, 1} g_{1}, \ldots, \omega_{i_{n}, n} g_{n}\right)=\left(\omega_{j_{(1 h)}, 1}, \ldots, \omega_{j_{(n h)}, n}\right) .
$$

Thus for every $t=1, \ldots, n$ the equality $\omega_{i_{t}, t} g_{t}=\omega_{j_{(t h)}, t}$ holds, and (3) implies that $i_{t}=j_{(t h)}$. Moreover, $\omega_{i, j}$ is a $G_{j}$-regular point for every $i, j$, so $g_{t}=e$ for $t=1, \ldots, n$, i.e., $g=h \in M$. Thus we obtain that

$\omega$ and $\theta$ are in the same $(G<M)$-orbit if and only if

$$
\text { there exist } h \in M \text { such that } \omega_{i_{t}, t}=\omega_{j_{(t h), t}} \text { for } t=1, \ldots, n \text {. }
$$

Now assume that $\omega$ and $\theta$ are chosen so that

$$
i_{t}=i_{s} \text { (respectively } j_{t}=j_{s} \text { ) if and only if } t, s \text { are in the same } P_{l},
$$

in particular, $\omega$ and $\theta$ are $(G<M)$-regular points. If $\omega$ and $\theta$ are in the same $(G>M)$-orbit, then (8) and (9) imply that $h$ permutes $P_{1}, P_{2}, P_{3}, P_{4}, P_{5}$. Since the order of a solvable subgroup of $\mathrm{Sym}_{5}$ is not greater than 24, we obtain that there exist at least $5\left(=\left|\operatorname{Sym}_{5}\right| / 24\right)$ points satisfying (9) and lying in distinct $(G>M)$-orbits.

We remark that the results in [2] and in the preceding papers are obtained by using probabilistic methods. In particular, given almost simple group $G$ with nonstandard action, it is shown that the probability for $k$ (where $k \geqslant \operatorname{Base}(G)$ ) randomly chosen points to form the base tends to 1 as $|G|$ tends to infinity.

The author is grateful to the referee for careful consideration of the paper and valuable comments. In particular, the referees' suggestions allows to simplify the proof of Corollary 2 . 


\section{References}

[1] L. Babai, A. J. Goodman, L. Pyber, Groups without faithful transitive permutation representations of small degree, Journal of Algebra, v. 195 (1997), 1-29.

[2] T.C. Burness, M.W. Liebeck, A.Shalev, Base sizes for simple groups and a conjecture of Cameron, Proc. London Math. Soc. (3), v. 98 (2009), 116162.

[3] J.H. Conway, R.T. Curtis, S.P. Norton, R.A. Parker, R.A. Wilson, Atlas of Finite Groups, Clarendon Press, Oxford, 1985.

[4] S. Dolfi, Large orbits in coprime actions of solvable groups, Trans. AMS, 2008. v. 360 (2008), $135-152$.

[5] R.M. Guralnick, J.S. Wilson, The probability of generating a finite soluble group, Proc. London Math. Soc. (3), v. 81 (2000), 405-427.

[6] B. Huppert, Endliche Gruppen I. Berlin: Springer, 1967. 808 p.

[7] I.M. Isaacs, Finite group theory. AMS Providence, RI: Amer. Math. Soc., 2008. 351 p.

[8] A. Seress, The minimal base size of primitive solvable permutation groups, J. London Math. Soc., v. 53 (1996), 243-255.

[9] E.P. Vdovin, Carter subgroups of finite almost simple groups, Algebra and Logic, v. 46 (2007), 90-119.

[10] E.P. Vdovin, Regular orbits of solvable linear $p^{\prime}$-groups, Sib. Electr. Math. Reports, v. 4 (2007), 345-360.

[11] E.P. Vdovin, V.I. Zenkov, On the intersection of solvable Hall subgroups in finite groups, Proc. Stekl. Inst. Math. Suppl. 3, 2009, 234-243.

[12] V.I. Zenkov, On the intersections of solvable Hall subgroups in finite nonsolvable groups, Tr. In-ta Matematiki i meckhaniki UrO RAN, v. 13 (2007), 86-89.

[13] The Kourovka notebook. Unsolved problems in group theory. Edited by V. D. Mazurov and E. I. Khukhro. 17-th. ed., Russian Academy of Sciences Siberian Division, Institute of Mathematics, Novosibirsk, 2010. 